\title{
PULMONARY MANIFESTATIONS OF LATE-ONSET SYSTEMIC LUPUS ERYTHEMATOSUS: A CASE REPORT
}

\author{
T. I. Kalenchic ${ }^{1}$, N. S. Didenko², S. L. Kabak ${ }^{3}$ \\ ${ }^{1}$ Associate Professor, Medical Rehabilitation and Physiotherapy Department, Belarusian State Medical University, Belarus \\ ${ }^{2}$ Center of Diagnostic and Rehabilitation of Gazprom transgaz, Russia \\ ${ }^{3}$ Professor, Human Morphology Department, Belarusian State Medical University, Belarus
}

\begin{abstract}
The current report presents a case of late-onset systemic lupus erythematosus (SLE). A 75-year-old Caucasian woman was admitted to the clinical hospital because of dyspnea, dry cough, lowgrade fever, wrist pain. There were no oral and skin lesions or lymphadenopathy observed. Laboratory tests revealed hypochromic microcytic anemia with hemoglobin $111 \mathrm{~g} / \mathrm{l}$, lymphopenia $0,54 \times 10 / \mathrm{l}$, the erythrocyte sedimentation rate (ESR) elevation up to $47 \mathrm{~mm} / \mathrm{h}$ and the C-reactive protein level up to 10,7 mg/l. Tumor markers (CA-125, CA-19.9, CA-15,3, $\alpha$-fetoprotein) concentration, hepatic and renal function were within the reference ranges. Of note, urinalysis didn't reveal proteinuria or microscopic hematuria and was considered normal. Computed tomography revealed bilateral pulmonary consolidation in S10, sacculated pleuritis, solitary lymphadenopathy, and pericardial effusions. Diagnosis of SLE was confirmed based on three clinical signs (synovitis of proximal interphalangeal joints, serositis including pleuritis, hematological disorders: anemia, lymphocytopenia) and positive findings of three immunological tests (anti-double-stranded DNA antibodies [Anti-dsDNA], antinuclear antibodies [ANA], and anti-nucleosome antibodies [ANUA]). This case demonstrates that late-onset SLE may be one of the reasons for the accumulation of pleural fluid in elderly patients.
\end{abstract}

Key words: pericarditis, pleuritis, late-onset systemic lupus erythematosus, serositis

\section{INTRODUCTION}

Systemic lupus erythematosus (SLE) is a complex chronic multisystem autoimmune disease of unknown etiology involving multiple organ systems that is associated with the antibody production and deposition of complement-fixing immune complexes $[1,2]$. SLE ranks among the top 20 leading causes of death in females aged 5-64 years.

The lowest incidences of SLE was reported in Africa and Ukraine (0.3/100 000 person-years), and the lowest prevalence - in Northern Australia ( 0 cases in a sample of 847 people) [3]. The age-adjusted incidence rate in women was five times higher than that for men (9.2 versus 1.8). Black women had an incidence rate of SLE nearly three times higher than Caucasian women [4]. In females, the peak age of incidence ranged from the third to seventh decades of life. For males, the peak incidence was failing mainly into the fifth to seventh decades [3]. Lateonset lupus (i.e., after the age of 50 years) typically manifests less disease activity, including less nephritis, and less organ damage yet more pulmonary manifestations [2]. However, the overall outcome is poorer in patients with late-onset disease, probably because of an increased comorbid condition.

The diagnosis of SLE in an elderly patient turns out to be challenging because of the insidious onset with non-specific symptoms and less common occurrence of severe manifestation, as well as presence co-existing chronic illnesses that may resemble SLE. Moreover, the less frequent prevalence of SLE in the elderly often results in late diagnosis [5]. The uncommon presentation, in turn, delays the initiation of the appropriate treatment.

We present a case of an elderly patient with the uncommon onset of the disease with challenging differential diagnosis. Written and signed consent to publish a case report was obtained from the patient.

\section{Case presentation}

A 75-year-old Caucasian woman was admitted to the Department of Pulmonology of the 6th Minsk clinical hospital having dyspnea, dry cough, lowgrade fever, wrist pain on January 1, 2019. There was no associated orthopnea, chest pain, pedal edema, or chills. She had no history of rash. The 
symptoms have gradually worsened for three weeks prior to admission. Moreover, she was hospitalized twice during 2018 with the diagnosis of communityacquired pneumonia and exudative pleuritis. The patient did not take medications that can cause lupuslike disease.

On admission, the vital signs were: body temperature $36,6^{\circ} \mathrm{C}$, blood pressure of $132 / 73 \mathrm{mmHg}$, pulse 70 beats per minute, respiratory rate 20 breaths per min and pulse oximetry of $94 \%$ on room air. Physical examination revealed pain and edema in the proximal interphalangeal (PIP) areas and wrist joints. There were no oral and skin lesions or lymphadenopathy observed. Cardiac examination disclosed a regular heart rhythm without any murmurs or gallop. The chest was clear to auscultation; the abdominal examination was negative for organomegaly or masses and there was no evidence of large synovial joints or leg edema.

Laboratory studies discovered hypochromic microcytic anemia with hemoglobin $111 \mathrm{~g} / \mathrm{l}$, lymphopenia $0,54 \times 10 \%$. The erythrocyte sedimentation rate (ESR) was elevated at $47 \mathrm{~mm} / \mathrm{h}$, the C-reactive protein (CRP) levels were elevated at $10,7 \mathrm{mg} / \mathrm{l}$, hepatic, and renal function were considered normal. Of note, urinalysis didn't show proteinuria or microscopic hematuria and was considered normal.

Chest computed tomography showed bilateral pulmonary consolidation in S10, sacculated pleuritis, solitary lymphadenopathy, and pericardial effusions (Fig 1A). Pulmonary embolism was ruled out. Wrist radiograph showed degenerative and inflammatory changes.

The pleural effusion was defined as exudative according to positive Rivalt's test and cytological examination (differential cell counts showed $8 \%$ neutrophils, $87 \%$ lymphocytes, and 5\% macro- phages). Bacterial culture tests, including mycobacteria, of the pleural effusion, were negative. The data obtained from the CT scan, gastroscopy, colonoscopy did not demonstrate any tumor mass. Tumor markers such as CA-125, CA-19.9, CA -15,3, $\alpha$-fetoprotein (AFP) were within the reference ranges. Therefore, infection and/or malignancy were concluded as an unlikely cause of the pleural and pericardial effusions.

Rheumatologic work-up results are presented in Table 1.

Table 1. Results of rheumatologic work-up

\begin{tabular}{|l|l|l|}
\hline Test & Actual result & Reference value/range \\
\hline Anti-dsDNA & $36,8 \mathrm{U} / \mathrm{ml}$ & $<10 \mathrm{U} / \mathrm{ml}$ \\
\hline ANA & Antibody Index 2,6 & $\begin{array}{l}\text { Negative Index }<1 \\
\text { Borderline Index 1.0-1.2 }\end{array}$ \\
\hline ANuA & $230 \mathrm{U} / \mathrm{ml}$ & $<20 \mathrm{U} / \mathrm{ml}$ \\
\hline Anti-CCP & $5,7 \mathrm{U} / \mathrm{ml}$ & $0-17 \mathrm{U} / \mathrm{ml}$ \\
\hline Anti MPO & $1,3 \mathrm{U} / \mathrm{ml}$ & $0-20 \mathrm{U} / \mathrm{ml}$ \\
\hline Anti PR3 & $1,6 \mathrm{U} / \mathrm{ml}$ & $0-20 \mathrm{U} / \mathrm{ml}$ \\
\hline RF & $2,2 \mathrm{U} / \mathrm{ml}$ & $<15 \mathrm{U} / \mathrm{mL}$ \\
\hline
\end{tabular}

Note: Anti-dsDNA = anti-double stranded DNA antibodies; ANA = antinuclear antibodies; ANuA = anti-nucleosome antibodies; anti-CCP = cyclic citrullinated peptide antibodies; Anti MPO = anti-myeloperoxidase antibodies; Anti PR3= anti-proteinase 3 antibodies; RF = rheumatoid factor

According to criteria of the Systemic Lupus International Collaborating Clinics (SLICC) the patient was diagnosed with SLE based on the presence of three clinical signs (synovitis of PIP joints, serositis including pleuritis, hematological disorders: anemia, lymphocytopenia) and three positive immunological tests (Anti-dsDNA, ANA, ANuA). At the same time, immunological markers of rheumatoid arthritis (anti-cyclic citrullinated peptide anti-
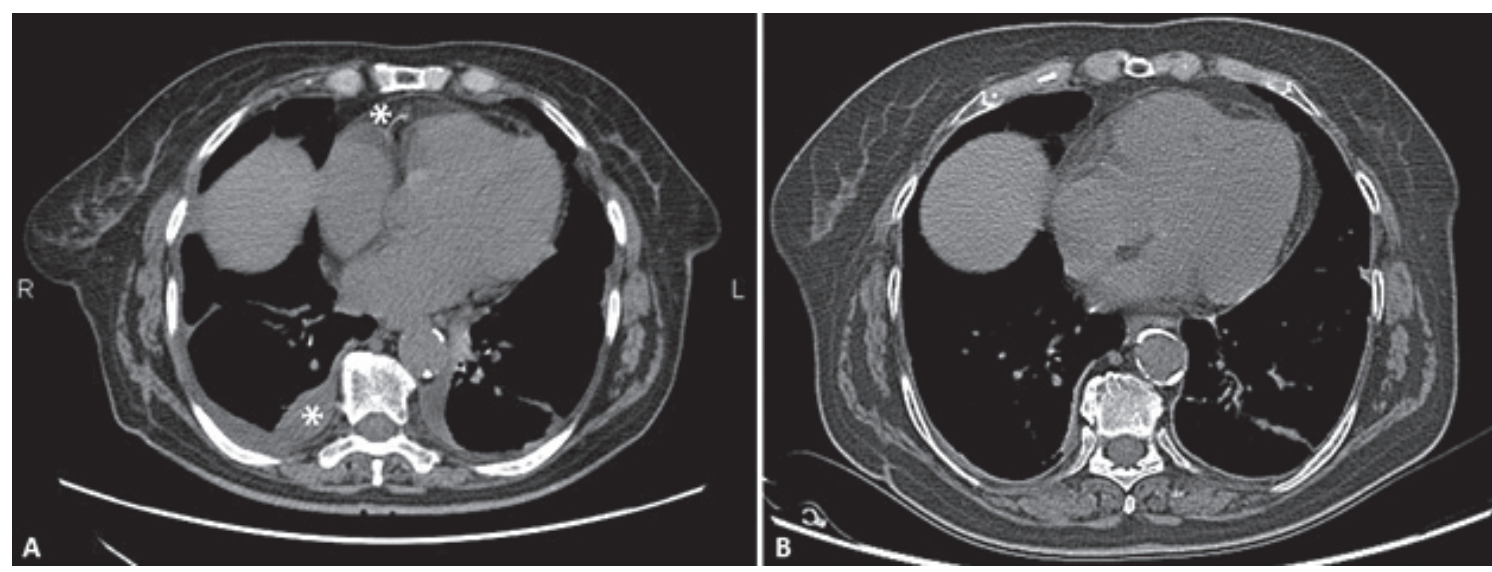

Fig. 1. Chest computed tomography (CT) axial view. A - January 2019; B - 5 months later. *shows the presence of pleural and pericardial effusions 
bodies [Anti CCP], rheumatoid factor) and vasculitis (anti-myeloperoxidase antibodies [Anti MPO], anti-proteinase 3 antibodies [Anti PR3]) were within the reference intervals.

Prednisolone therapy (60 mg IV daily) was initiated upon the confirmation of the diagnosis started, and the patient was discharged from the hospital with significant improvement of the respiratory symptoms after two weeks of the active treatment.

After five months, a CT computer tomography of the chest and immunological tests were performed. The presence of fluid in the pleural and pericardial space was not found (Fig. 1B). The level of the lupus anticoagulant was within the reference interval.

\section{Discussion}

Like other autoimmune diseases, SLE predominantly affects women with a high incidence during childbearing age and steeply declines after menopause. Numerous studies report an increased frequency of SLE in the non-Caucasian population with 5 to 9-fold increased incidence and 2- to 3-fold increased prevalence in certain ethnicities such as African-Caribbean and South/East Asians compared to Caucasians [1].

The current report represents a case of a 75 -year-old Belarusian woman diagnosed with SLE six months after the appearance of pleuritis, which was considered to be the first clinical sign of the disease. To date, there have been only six cases of SLE with pleural effusion as the initial manifestation reported in the English literature6

Clinical manifestations of late-onset SLE have been elucidated from early-onset SLE. Serositis, interstitial pneumonia, and Sjogren's syndrome are frequently linked to late-onset SLE, while cutaneous manifestation, photosensitivity, arthritis, and nephritis occur less frequently. Elderly patients are frequently admitted to the hospital with nonspecific symptoms such as fatigue, weight loss, and acute kidney injury [7]. In the present case, in addition to exudative pleurisy and pericarditis, the patient had one of such musculoskeletal manifestations of SLE - unexpressed arthralgia of the small joints of the hand. Non-specific symptoms may be a reason for SLE being misdiagnosed [1].

A systematic literature review and meta-regression confirmed the very high sensitivity of anti-nuclear antibodies (ANA) for SLE [8]. We used this immunological test to verify the diagnosis. However, a major drawback of ANA is the low diagnostic specificity since a positive test can be found in numerous autoimmune diseases (autoimmune thyroiditis, autoimmune liver diseases, and myasthenia among others) as well as in healthy individuals especially at low titers [1]. Positive ANAs are common, especially in unwell elderly individuals. Thus, a positive ANAs are of neither specific nor prognostic value, as most people with these antibodies will never develop lupus or any other autoimmune or inflammatory disease [9].

Some other specific autoantibodies were used to confirm the diagnosis of SLE. Specifically, antidouble-stranded DNA and anti-nucleosome antibodies which are highly specific for SLE and useful to assess the disease activity, were utilized [10]. These tests were positive in the current case. Often in late-onset SLE anti-double-stranded DNA test tends to yield negative results 7 while some of the other studies suggested that Anti-dsDNA is less frequent in late-onset SLE, some other studies showed that there is no difference between the age groups [11]. Compared to early-onset SLE, rheumatoid factor antibody test yields positive results more often in late-onset SLE. In the current observation, the rheumatoid factor antibody concentration was within the reference range.

\section{Conclusion}

This case demonstrates that late-onset SLE may be one of the reasons for the accumulation of pleural fluid in elderly patients.

\section{References}

1. Gergianaki I, Bertsias G. Systemic Lupus Erythematosus in Primary Care: an update and practical messages for the General Practitioner. Front Med (Lausanne). 2018, 5, 161. doi: 10.3389/fmed.2018.00161. eCollection 2018

2. Gergianaki I, Bortoluzzi A, Bertsias G. Update on the epidemiology, risk factors, and disease outcomes of systemic lupus erythematosus. Best Pract Res Clin Rheumatol. 2018, 32(2), 188-205.

3. Rees F, Doherty M, Grainge MJ, Lanyon P, Zhang W. The worldwide incidence and prevalence of systemic lupus erythematosus: a systematic review of epidemiological studies. Rheumatology (Oxford). 2017, 56(11), 1945-1961.

4. Lim SS, Bayakly AR, Helmick CG, Gordon C, Easley KA, Drenkard $\mathrm{C}$. The incidence and prevalence of systemic lupus erythematosus, 2002-2004: The Georgia Lupus Registry. Arthritis Rheumatol. 2014, 66(2), 357-368.

5. Ugolini-Lopes MR, Santos LPS, Stagnaro C, Seguro LPC, Mosca M, Bonfá E. Late-onset biopsy-proven lupus nephritis without other associated autoimmune diseases: severity and long-term outcome. Lupus. 2019, 28(1), 123-128.

6. So C, Imai R, Tomishima Y, Nishimura N. Bilateral pleuritis as the initial symptom of systemic lupus erythematosus: a 
case series and literature review. Intern Med. 2019;58(11), 1617-1620.

7. Kutky M, Aloudat S. Late-onset systemic lupus erythematosus with lupus nephritis in a 74-year-old male: a brief case and review. Can J Kidney Health Dis. 2018; 5, 2054358118793397.

8. Leuchten N, Hoyer A, Brinks R, Schoels M, Schneider M, Smolen Jet al. Performance of antinuclear antibodies for classifying systemic lupus erythematosus: a systematic literature review and meta-regression of diagnostic data. Arthritis care \& research.2018, 70(3), 428-438.

9. Berens-Norman HM, Boackle SA. Editorial: subduing lupus: can preclinical autoimmune disease be arrested? Arthritis Rheumatol. 2016, 68(10), 2357-2360.

10. Živković V, Stanković A, Cvetković T, Mitić B, Kostić S, Nedović $\mathrm{J}$ et al. Anti-dsDNA, anti-nucleosome and anti-C1q antibodies as disease activity markers in patients with systemic lupus erythematosus. Srp Arh Celok Lek. 2014, 142(7-8), 431-436.

11. Cildag S, Kara Y, Cakir E, Cildag MB, Senturk T. Comparison of clinical and laboratory findings in patients with systemic lupus erythematosus with regard to age at onset. Eurasian $\mathrm{J}$ Med. 2019, 51(1), 17-21

Submitted: 16.01.2020

$\triangle$ Corresponding author:

S. L. Kabak Human Morphology Department,

Belarusian State Medical University

83 Dzerzhinsky Ave.

Minsk, Belarus 220116

Phone: +375296588339

e-mail: kabakmorph@gmail.com 